\title{
In-orbit performance of the Herschel/ SPIRE imaging Fourier transform spectrometer
}

Naylor, David, Baluteau, Jean-Paul, Barlow, Mike, Benielli, Dominique, Ferlet, Marc, et al.

David A. Naylor, Jean-Paul Baluteau, Mike J. Barlow, Dominique Benielli, Marc Ferlet, Trevor R. Fulton, Matthew J. Griffin, Timothy Grundy, Peter Imhof, Scott Jones, Ken King, Sarah J. Leeks, Tanya L. Lim, Nanyao Lu, Gibion Makiwa, Edward T. Polehampton, Giorgio Savini, Sunil D. Sidher, Locke D. Spencer, Christian Surace, Bruce M. Swinyard, Roger Wesson, "Inorbit performance of the Herschel/SPIRE imaging Fourier transform spectrometer," Proc. SPIE 7731, Space Telescopes and Instrumentation 2010: Optical, Infrared, and Millimeter Wave, 773116 (5 August 2010); doi: 10.1117/12.856299

Event: SPIE Astronomical Telescopes + Instrumentation, 2010, San Diego, California, United States 


\title{
In-orbit performance of the Herschel/SPIRE imaging Fourier transform spectrometer
}

\author{
David A. Naylor ${ }^{*} a$, Jean-Paul Baluteau ${ }^{b}$, Mike J. Barlow ${ }^{c}$, Dominique Benielli ${ }^{b}$, Marc Ferlet ${ }^{d}$, Trevor \\ R. Fulton ${ }^{e}$, Matthew J. Griffin ${ }^{f}$, Timothy Grundy ${ }^{d}$, Peter Imhof ${ }^{e}$, Scott Jones ${ }^{a}$, Ken King ${ }^{d}$, Sarah J. \\ Leeks $^{d}$, Tanya L. Lim ${ }^{d}$, Nanyao Lu ${ }^{g}$, Gibion Makiwa ${ }^{a}$, Edward T. Polehampton ${ }^{a}$, Giorgio Savini ${ }^{c}$, \\ Sunil D. Sidher ${ }^{d}$, Locke D. Spencer ${ }^{f}$, Christian Surace $^{b}$, Bruce M. Swinyard $^{d}$, Roger Wesson $^{b}$ \\ ${ }^{a}$ Institute for Space Imaging Science, University of Lethbridge, Alberta, T1K 3M4, Canada \\ ${ }^{b}$ Laboratoire d'Astrophysique de Marseille, Marseille, France, 13388 \\ ${ }^{c}$ Department of Physics and Astronomy, University College London, UK \\ d. Space Science and Technology Department, Rutherford Appleton Laboratory, OX11 0QX, UK \\ ${ }^{e}$ Blue Sky Spectroscopy Inc., 9 - 7404 Avenue South, Lethbridge, Alberta, T1J 0N9, Canada \\ ${ }^{f}$ School of Physics and Astronomy, Cardiff University, Cardiff, United Kingdom, CF24 3AA, UK \\ ${ }^{g}$ National Herschel Science Center, California Institute of Technology, Pasadena, USA, 91125
}

\begin{abstract}
The Spectral and Photometric Imaging Receiver (SPIRE) is one of three scientific instruments onboard the European Space Agency's Herschel Space Observatory launched on 14 May 2009. The low to medium resolution spectroscopic capability of SPIRE is provided by an imaging Fourier transform spectrometer of the Mach-Zehnder configuration. Results from the in flight performance verification phase of the SPIRE spectrometer are presented and conformance with the instrument design specifications is reviewed.
\end{abstract}

Keywords: Herschel, SPIRE, imaging Fourier transform spectrometer, characterization

\section{INTRODUCTION}

The Spectral and Photometric Imaging Receiver (SPIRE) is one of three scientific instruments onboard the European Space Agency's Herschel Space Observatory launched on 14 May $2009^{1}$. SPIRE contains a three-band imaging photometer operating at 250,350 and $500 \mu \mathrm{m}$, and an imaging Fourier Transform Spectrometer (FTS), which covers the wavelength range of 194-671 $\mu \mathrm{m}\left(447-1550 \mathrm{GHz} ; 14.9-51.5 \mathrm{~cm}^{-1}\right)^{2}$. The SPIRE instrument package is approximately $700 \times 400 \times 400 \mathrm{~mm}$ in size and is supported from the 10-K Herschel optical bench by thermally insulating mounts. The photometer and spectrometer are mounted on opposite sides of the SPIRE optical bench, which contains the optics, detector arrays (three for the photometer, and two for the spectrometer), an internal ${ }^{3} \mathrm{He}$ cooler to provide the required detector operating temperature of $\sim 0.3 \mathrm{~K}$, filters, mechanisms, internal calibrators, and housekeeping thermometers. Both the photometer and spectrometer have cold pupil stops conjugate with the Herschel secondary mirror, which is the telescope system pupil, and defines an effective 3.29-m diameter primary aperture. All five detector arrays use hexagonally close-packed, conical feedhorn-coupled spider-web neutron transmutation doped Germanium bolometers ${ }^{3}$. The bolometers are AC biased with frequency adjustable between 50 and $200 \mathrm{~Hz}$, avoiding $1 / f$ noise from the cold JFET readout readouts.

\section{SPIRE SPECTROMETER}

A detailed description of the SPIRE spectrometer is given elsewhere ${ }^{2,45}$, the main features are presented here. The SPIRE spectrometer is of the Mach-Zehnder configuration and uses two broadband intensity beam splitters ${ }^{6}$ to provide spatial separation of the input and output ports. One input port views a 2.6' diameter field of view on the sky and the other an on-board reference source. Two bolometer arrays at the output ports cover overlapping bands of 194-313 $\mu \mathrm{m}$ (SSW) and 303-671 $\mu \mathrm{m}$ (SLW). As with any FTS, each scan of the moving mirror produces an interferogram in which

* David A Naylor: E-mail: naylor@uleth.ca, Telephone: 14033292426

Space Telescopes and Instrumentation 2010: Optical, Infrared, and Millimeter Wave, edited by Jacobus M. Oschmann Jr., Mark C. Clampin, Howard A. MacEwen, Proc. of SPIE Vol. $7731,773116 \cdot$ ? 2010 SPIE · CCC code: 0277-786X/10/\$18 · doi: 10.1117/12.856299 
the spectrum of the entire band is encoded with the spectral resolution corresponding to the maximum mirror travel. A schematic of the SPIRE spectrometer is shown in Figure. 1.

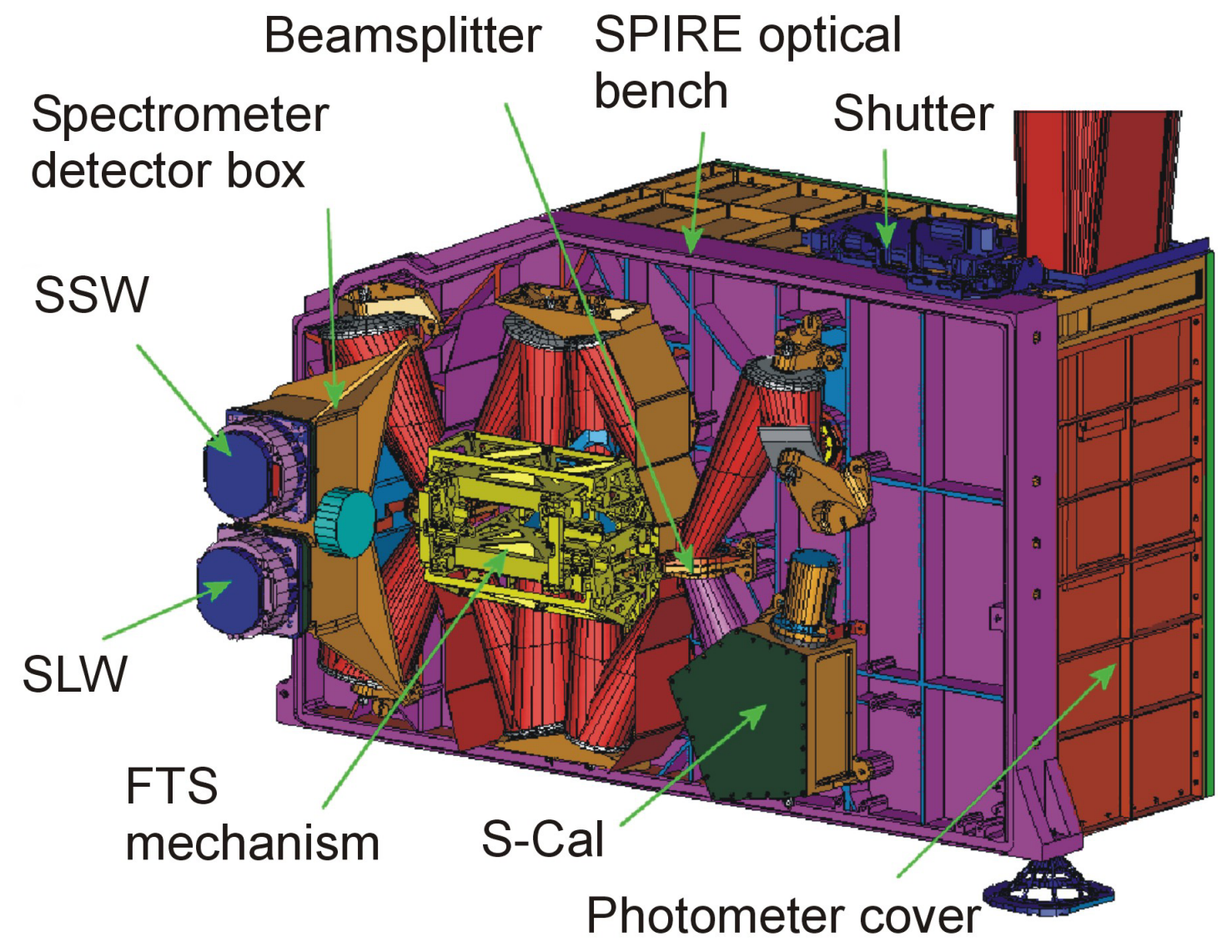

Figure 1. A schematic of the imaging Fourier transform spectrometer side of the SPIRE optical bench showing the key components.

A single, back-to-back scanning roof-top mirror assembly simultaneously modulates the optical path in both interferometer arms. The mechanism uses a double parallelogram linkage with flex pivots and is essentially frictionless. Metrology information is provided by a Moiré fringe encoder. The two hexagonally close-packed spectrometer arrays contain 19 feedhorn coupled detectors in the long-wavelength array (SLW) and 37 in the short-wavelength array (SSW), shown schematically in Fig. 2. The feedhorn coupled architecture was chosen as the best option given the achievable sensitivity, the requirements for the largest possible field of view and high stray light rejection, and limitations on the number of detectors imposed by spacecraft resource budgets. The detector feedhorns are designed for maximum efficiency, requiring an entrance aperture close to $2 F \lambda$, where $F$ is the final optics focal ratio of the spectrometer and $\lambda$ is the wavelength. Quasi-optical filters ${ }^{6}$ located in front of each detector array at the two output ports, together with the cutoff wavelengths of the feedhorn output waveguides, define the spectral range of the two overlapping FTS bands.

While the feedhorns for the SPIRE photometer are single-moded ${ }^{2}$ and thus provide close to Gaussian illumination of the telescope pupil, in the case of the spectrometer, the requirement to provide good sensitivity across the broad wavelength range results in the feedhorns being multi-moded with correspondingly more complex illumination of the pupil. The SSW feedhorns are sized to give $2 F \lambda$ pixels at $225 \mu \mathrm{m}$ and the SLW horns are $2 F \lambda$ at $389 \mu \mathrm{m}$. This arrangement has the advantage that there are many co-aligned pixels in the combined field of view (shaded in Figure 2). The SSW beams on the sky are 33" apart, and the SLW beams are separated by $51 "$. 
A thermal source, $\mathrm{SCal}^{7}$, located at the second input port of the spectrometer is available to allow the interferometric modulation from the background power from the telescope to be nulled, thereby reducing the dynamic range of the resulting interferogram at the zero path difference position of the interferometer.
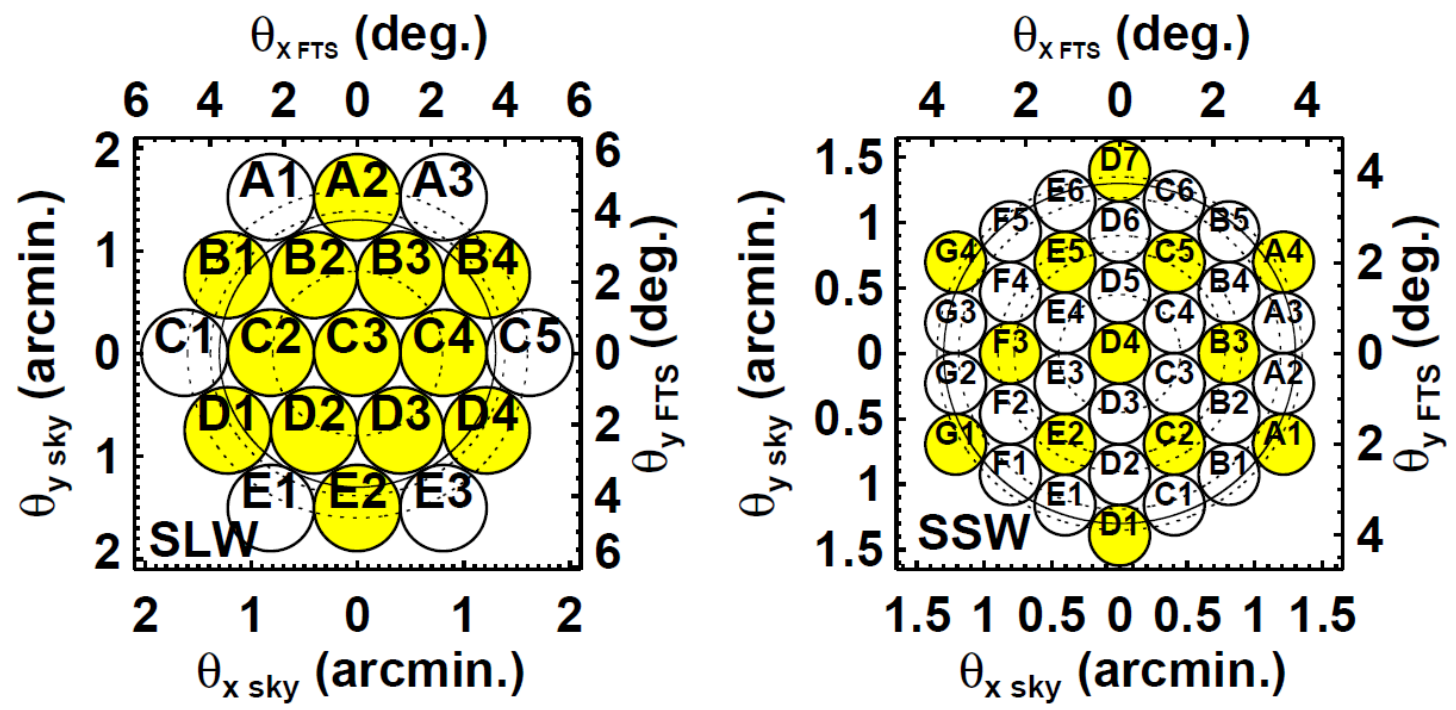

Figure 2. The angular position of SLW \& SSW detectors on the sky (left and bottom axes) and the divergence angle within the FTS (top and right axes) for both detector arrays. The concentric dashed circles represent the off-axis rings of HCP detectors in each array and are used in the obliquity analysis as discussed in section 3.3. The shaded pixels represent those that are co-aligned in both arrays. The solid circle represents the extent of the SPIRE IFTS 2.6' unvignetted FOV, outside of which partial vignetting occurs.

For an FTS the spectral resolution element is given by $1 /(2 L)$ where $L$ is the maximum optical path difference between the interfering beams. In the case of SPIRE, the maximum scan length is $3.5 \mathrm{~cm}$, corresponding to an optical path difference of $14 \mathrm{~cm}$. Three values of spectral resolution have been adopted as standard: high, medium, and low, giving unapodised resolutions of $0.04,0.24$, and $0.83 \mathrm{~cm}^{-1}(1.2,7.2$, and $25 \mathrm{GHz})$, respectively. The highest unapodised resolution available is $0.04 \mathrm{~cm}^{-1}(1.2 \mathrm{GHz})$, which corresponds to a FWHM of the instrument spectral response function of $0.048 \mathrm{~cm}^{-1}$. For this resolution, the resolving power $\lambda / \Delta \lambda$ varies between 1300 and 370 at the short and long wavelength extrema, respectively. The standard unapodised resolution adopted for the lower resolution spectrophotometric operating mode is $0.83 \mathrm{~cm}^{-1}$, for which $\lambda / \Delta \lambda$ varies between 20 and 60 over the wavelength range.

The standard operating mode for the FTS is to scan the mirror mechanism at constant speed (nominally $0.5 \mathrm{~mm} / \mathrm{s}$ ) with the telescope pointing fixed, providing an optical path rate of change of $2 \mathrm{~mm} / \mathrm{s}$ due to the factor of four folding in the Mach-Zehnder design. The resulting electrical modulation frequencies of the astronomical source lie in the range of 3-10 Hz. The AC-biased bolometer signals are de-modulated by individual lock-in amplifiers (LIAs). Each LIA comprises a bandpass filter and a square wave demodulator, followed by a $25-\mathrm{Hz}$ low-pass filter which is matched to the modulation frequencies produced by the FTS. Finally the detector signal is sampled at $80 \mathrm{~Hz}$, a rate which can be transmitted to Earth with no on-board processing.

\section{IN-FLIGHT PERFORMANCE}

Following a successful launch, the commissioning and performance verification phases of the mission revealed that all SPIRE subsystems were fully functional and met their key requirements. Highlights include: the ${ }^{3} \mathrm{He}$ cooler has a holdtime in excess of $46 \mathrm{hrs}$, allowing two full days of SPIRE operation after a cooler cycle. The operating temperature of the two FTS detector arrays is $286+/-1 \mathrm{mK}$. This value is in line with ground-based measurements and slightly lower than used in the pre-launch instrument sensitivity model. Remarkably only two of the spectrometer detectors are unusable. The radiant background loading on the detectors, from the thermal emission of the Herschel telescope itself, which at $\sim 85 \mathrm{~K}$ is the dominant source of emission, and any additional stray light, is consistent with, but lower than the values used in pre-launch estimation of the instrument performance. The lower radiant background from the Herschel 
telescope allows the FTS to be operated without the need for the modulation nulling source, Scal, which essentially reduces the overall photon noise by a factor of $\sqrt{ } 2$. It is clear that the launch of Herschel not only proved to be a rather benign event for the SPIRE instrument, but also that the operational conditions have transpired to make the spectrometer more sensitive than predicted. The following sections detail key aspects of the in-flight spectrometer performance and are compared with the pre-flight predictions ${ }^{8}$.

\subsection{Spectral range}

The SPIRE FTS was designed to cover the spectral range $200-670 \mu \mathrm{m}$ to match the emission peak of continuum and line emission from the cold interstellar medium. This range is also covered by the Herschel/HIFI instrumentc ${ }^{9}$ and has a small overlap with the long wavelength end of the Herschel/PACS instrument ${ }^{10}$. The two bands of the SPIRE FTS were designed to have a small overlap to simplify cross-calibration while maximizing the spectral range. The spectral bandpass is defined as the points where the spectral intensity reaches half of its average in-band value. The high pass edges being determined by the exit apertures of the conical feedhorns, while the low pass edges are determined by the optical filters placed immediately in front of the detector arrays. The spectral range as measured in-flight is shown in the Figure 3 for the central pixels of each array. The in-flight values are in agreement with the design requirements as shown in Table 1.
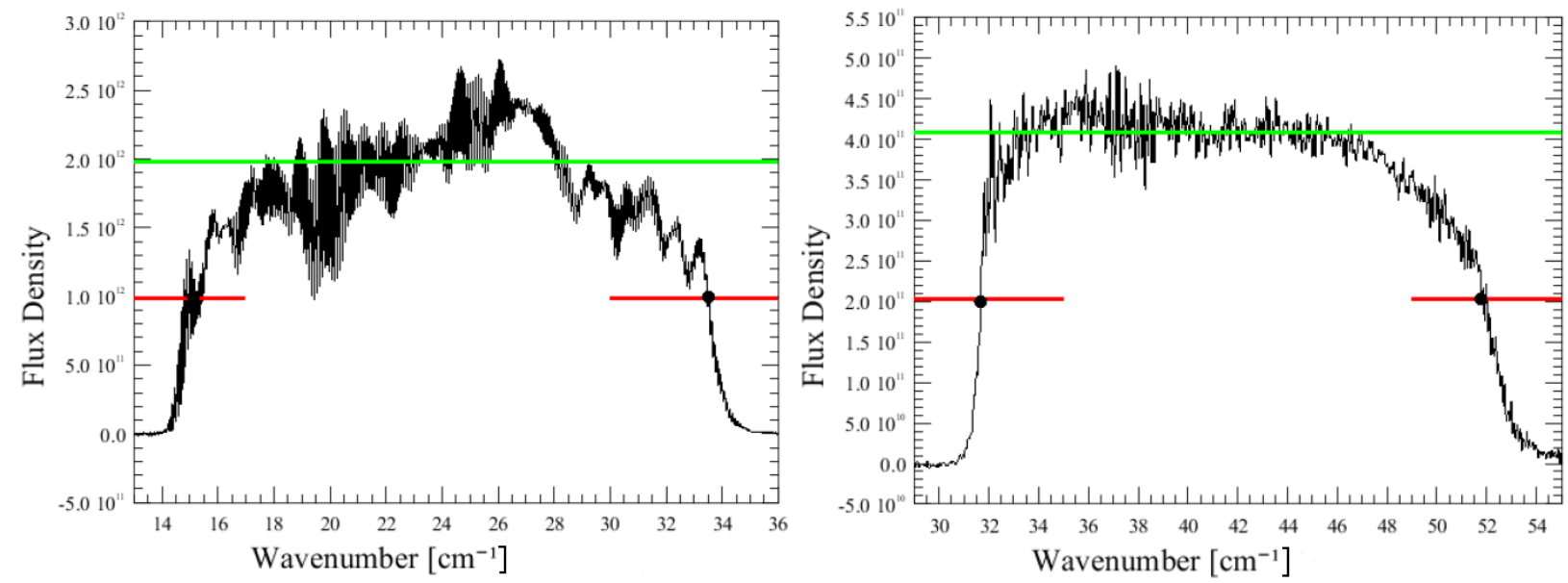

Figure 3. Spectral range of the central pixels of each array SLWC3 (left) and SSWD4 (right) determined from in-flight measurements.

Table 1. Comparison of goal and measured spectral range.

\begin{tabular}{|c|c|c|}
\hline Band & Requirement & Spectral Range \\
& In-Flight Measurement \\
\hline SLW & $14.9-33.3 \mathrm{~cm}^{-1}(671-300 \mu \mathrm{m})$ & $14.9-33.0 \mathrm{~cm}^{-1}(671-303 \mu \mathrm{m})$ \\
\hline SSW & $33.3-50.0 \mathrm{~cm}^{-1}(300-200 \mu \mathrm{m})$ & $32.0-51.5 \mathrm{~cm}^{-1}(312.5-194 \mu \mathrm{m})$ \\
\hline
\end{tabular}

\subsection{Instrumental line shape (ILS)}

In the case of an ideal Fourier transform spectrometer, the instrumental line shape is given by the cardinal sine or sinc function:

$$
I L S(\sigma)=2 L \sin (2 \pi \sigma L) / 2 \pi \sigma L
$$

where $\mathrm{L}$ is the maximum optical path difference created by the scan mirror travel and $\sigma$ is the wavennumber in $\mathrm{cm}^{-1}$. The sinc function has a full width at half maximum (FWHM) of $\sim 0.603 / L$ and is characterized by a series of secondary lobes of slowly decreasing amplitude, the amplitude of the first minimum being $-21.7 \%$ of the main lobe. Using a technique known as apodization it is possible post hoc to decrease the amplitudes of the sidelobes associated with the sinc function 
at the cost of increasing the FWHM of the ILS (i.e., decreasing the spectral resolution) ${ }^{11}$. However, the accuracy of derived spectral line parameters can be improved if the information contained in the extended secondary lobes is used in the subsequent data analysis.

To study the ILS requires measurements of an unresolved spectral line(s). During ground based testing the monochromatic output from a molecular laser and a photomixer were used ${ }^{12}$. In-flight it has proven possible to utilize the strong rotational line emission from the CO molecule to this end. There are $10{ }^{12} \mathrm{CO}$ lines within the SPIRE FTS band. These lines are relatively isolated and, by removing a continuum baseline, it is possible to extract the ILS from each. Figure 4 shows one example of a SPIRE spectrum of the Orion bar ${ }^{13}$.

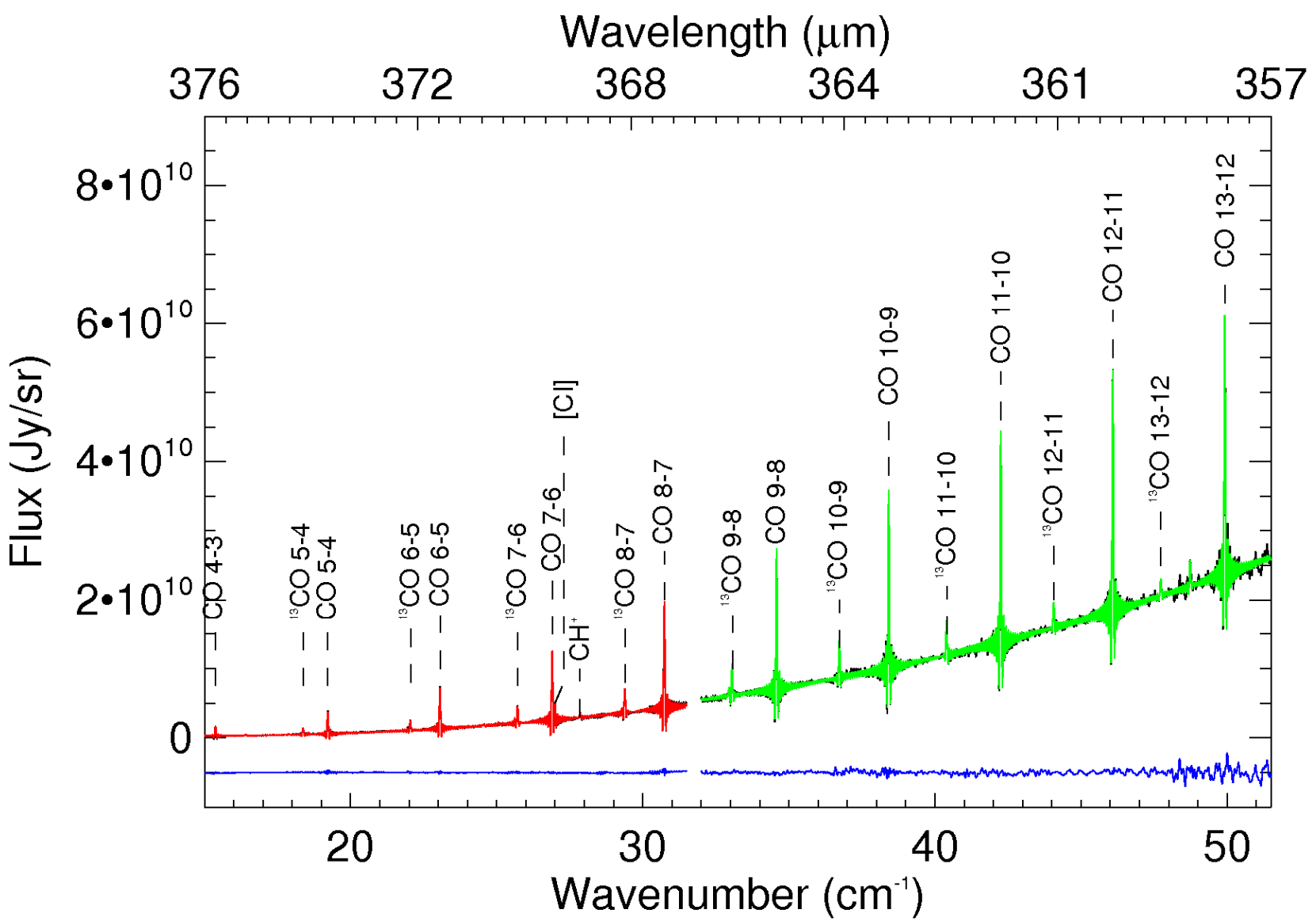

Figure 4. SPIRE spectrum of the Orion bar showing the plethora of ${ }^{12} \mathrm{CO}$ and ${ }^{13} \mathrm{CO}$ lines on a well defined continuum. The black line is a model fit to the SLWC3 (red) and SSWD4 (green) spectra. The lower trace shows the residual spectrum to the same scale.

The in-flight ILS has been established by performing a weighted average of the baseline removed CO line spectrum for nine of the ${ }^{12} \mathrm{CO}$ lines in spectra similar to that shown in Figure 4 (omitting the CO 7-6 line which lies close to the CI line at $370 \mu \mathrm{m}(809.345 \mathrm{GHz}))$, in two galactic sources, with data obtained in five different observations. Over 1000 individual line spectra are included in this analysis. The resulting normalized ILS is shown in Figure 5. While the forward and reverse scans of the interferometer have been processed independently, the differences between the measured profiles is barely discernable. There is however, a slight difference between the recovered ILS and the classical sinc function, particularly in the region of the first negative high frequency lobe.

The observed asymmetric line shape may be due in part to the effect of the finite entrance aperture and optical alignment within the interferometer, commonly referred to as the obliquity effect, which results in deviations from the ideal sinc $\mathrm{ILS}^{14}$, and is particularly important for off axis pixels. Since advanced line fitting routines require a detailed knowledge of the ILS in order to retrieve accurate spectral information (e.g. line position, amplitude and half-width), deviations from the ideal sinc ILS, caused by the obliquity effect, must be taken into account. If left uncorrected, spectral features will exhibit an apparent shift in line centre, an increase in linewidth and a loss of symmetry. The top row in Figure 6 
shows the superimposed CO line spectra of several pixels in the SLW and SSW bands, uncorrected for the effects of obliquity. The spectra from off-axis pixels are shifted to longer wavelengths. The bottom row shows the identical, obliquity corrected spectra, which are now correctly registered. The closely separated CO 7-6 and CI lines are now clearly evident, as is the fundamental rotational transition of $\mathrm{CH}^{+15}$.

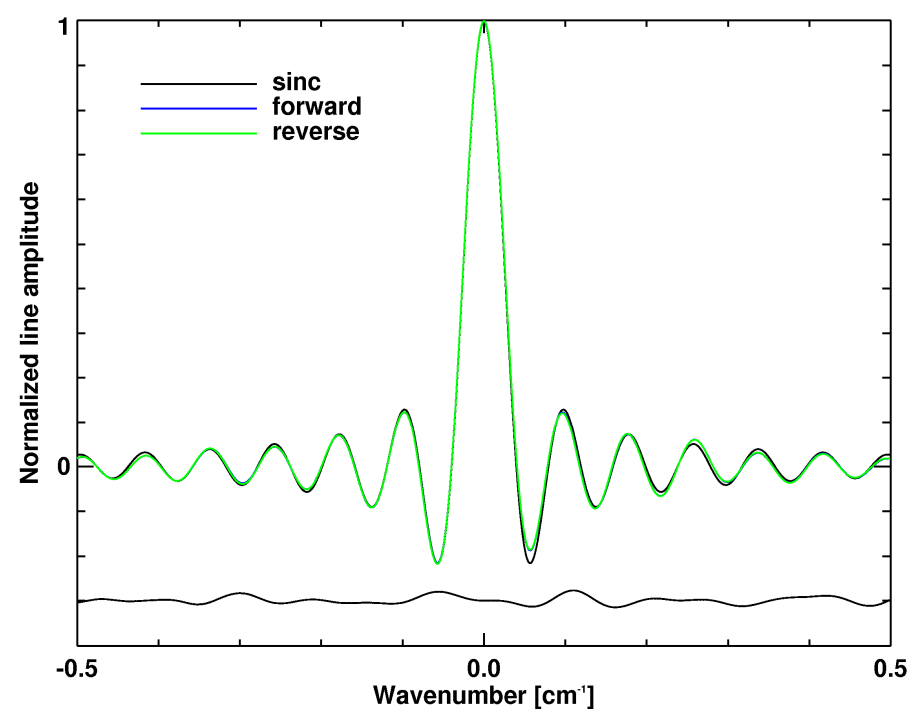

Figure 5. Normalized ILS derived from over 1000 individual ${ }^{12} \mathrm{CO}$ line measurements. The difference between forward (green) and reverse (blue) ILS is hardly discernable. The classical sinc function (black) shows small deviations close to the first negative high frequency lobe. The lower trace shows difference to the same scale.

The high quality and stability of the ILS evident in Figure 5 together with a thorough understanding of the obliquity effects demonstrated in Figure 6 will be exploited in the extraction of spectral line parameters for both arrays, through line fitting and deconvolution techniques currently under development. One example of spectral line fitting to the spectrum obtained with the central pixel SLWC3 (Figure 4) is shown in Figure 7.
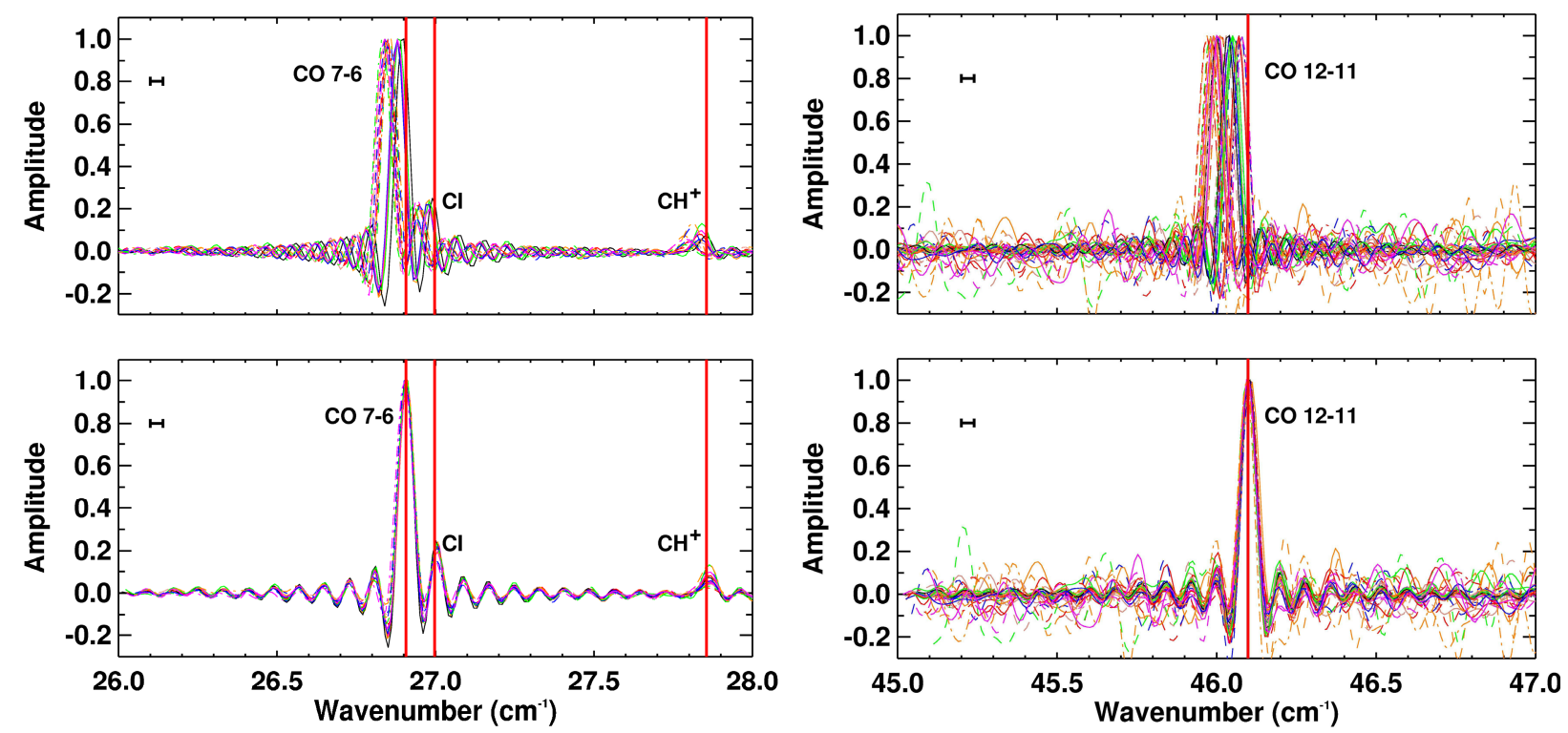

Figure 6. In the top row spectra from several pixels, uncorrected for the effects of obliquity, for a CO transition in the SLW (left) and SSW (right) band have been superimposed. The bottom row shows the identical, obliquity corrected spectra. The bar in each figure represents the spectral resolution element. 


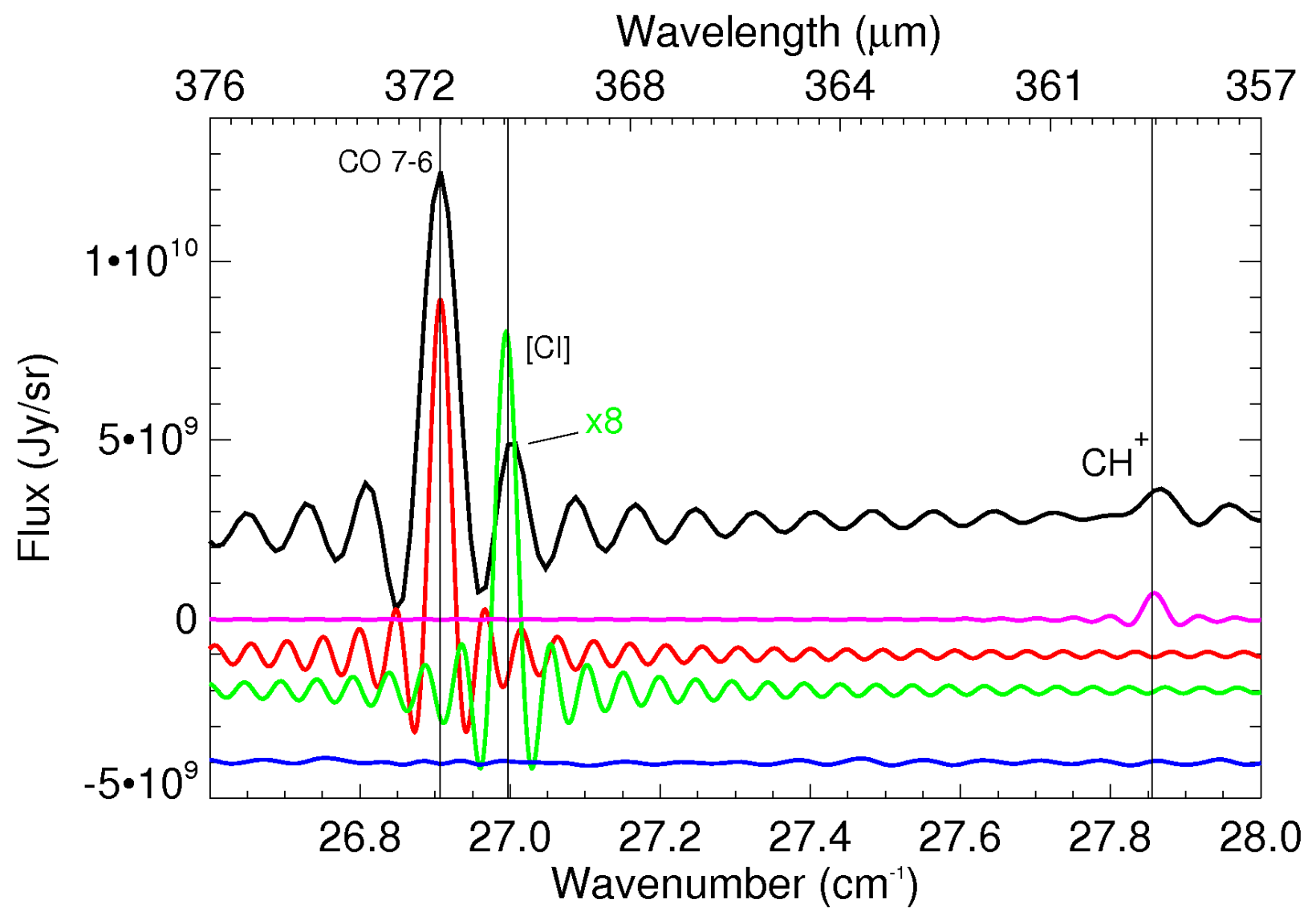

Figure 7. Results of spectral line fitting using the ILS of the central pixel SLWC3 applied to the spectrum of Figure 4 (black). Individual components retrieved from the fitting procedure: the partially resolved CO 7-6 (red) and CI (green x8) lines, and the fundamental rotational transition of $\mathrm{CH}+$ (purple), the latter observed for the first time with the SPIRE FTS ${ }^{15}$. The vertical lines show the rest frame frequencies of, and the lower trace shows the residual after line fitting to, the three species.

\subsection{Wavelength accuracy}

For an infinitely small detector located on the optical axis, the wavelength scale of an FTS is uniquely determined by the metrology system. For detectors of finite size and off-axis, the scale must be corrected using the obliquity analysis described above. In the case of the SPIRE FTS, the wavelength scale accuracy has been verified using line fits to the ${ }^{12} \mathrm{CO}$ lines in five Galactic sources assuming the theoretical (i.e., sinc) instrumental line shape. In agreement with other practitioners ${ }^{16}$ we find that the line centres can be determined to within a small fraction $(<1 / 20$ th $)$ of the spectral resolution element if the signal-to-noise is high (Figure 8). Further, we find very good agreement between observations of different galactic sources and across both SLW and SSW bands. The spectral resolution measured in-flight for the three nominal spectroscopic modes of the SPIRE FTS are summarized in Table 2.

Table 2. Measured in-flight spectral resolution $\left(\mathrm{cm}^{-1}\right)$ for the three nominal SPIRE spectroscopic observing modes.

\begin{tabular}{|c|c|c|}
\hline Mode & Spectral Resolution & Line FWHM \\
\hline High & $0.0398 \pm 0.0002$ & $0.0480 \pm 0.0002$ \\
\hline Medium & $0.24 \pm 0.01$ & $0.29 \pm 0.012$ \\
\hline Low & $0.83 \pm 0.04$ & $1.0 \pm 0.05$ \\
\hline
\end{tabular}




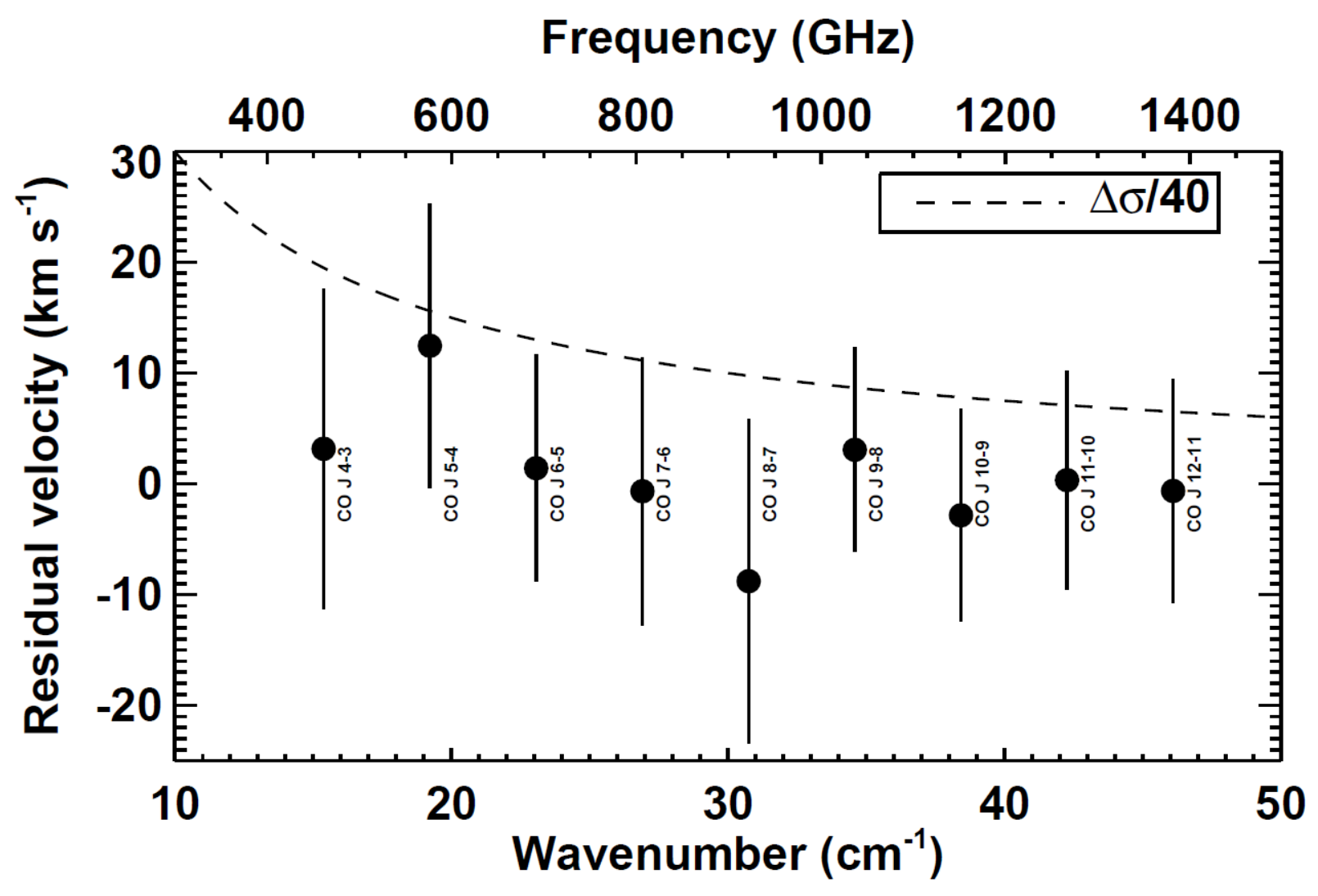

Figure 8. The wavelength accuracy expressed in terms of residual velocity, for the CO lines observed in Orion over both the SLW and SSW arrays. In determining the residual velocity, the effects of obliquity and of Doppler motion of the source and Herschel telescope have been removed. The dots show the mean residual velocity with $1-\sigma$ error bars. For reference the dashed curve shows one fortieth of the spectral resolution element of the SPIRE FTS.

\subsection{Beam profiles}

The broad spectral coverage of the two FTS bands precludes the use of a single-moded feedhorn. The resulting wavelength dependent illumination of the telescope pupil by the different modes that couple to the conical feedhorns is non-trivial. A detailed knowledge of the variation of the spectrometer beams as a function of wavelength, however, is required in order to calibrate the resulting spectra. The spectrometer beam shape has been determined in-flight by taking medium resolution spectra of Neptune, which approximates a point source, while the planet was stepped across the focal plane. An 11 x 11 grid of spectra taken with a step size of 9" provided a data cube for the central detector pair (SLWC3/SSWD4) that has been analyzed to yield an estimate of the wavelength dependent beam profile. Figure 9 shows the surface plots resulting from least squares of fitting the raw data with 2-dimensional elliptical Gaussian and Airy profiles respectively. The raw spectral data are shown in the left column at frequencies of $20,30,40$ and $50 \mathrm{~cm}^{-1}$ respectively. The 2-dimensional elliptical Gaussian and Airy best fits and the residuals are shown in subsequent columns. While neither function is expected to describe accurately the complex multi-moded beam and further, that the beam is marginally Nyquist sampled at the shortest wavelengths (the time required to acquire spectral maps on a fine grid being prohibitive), the results are nonetheless instructive. Both the elliptical Gaussian and Airy fits return similar values for the beam size and ellipticity as shown in Figure 10 and Table 3. Moreover, the wavelength dependence agrees well with the expected mode onset of the feedhorns ${ }^{17}$ at 19, 25, 31 and $41 \mathrm{~cm}^{-1}$ respectively. 


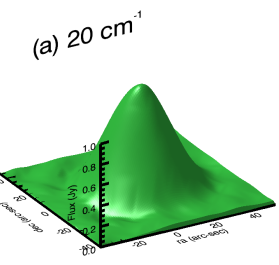
(b) Gaussian

(c) Residual

(d) Airy

(e) Residual
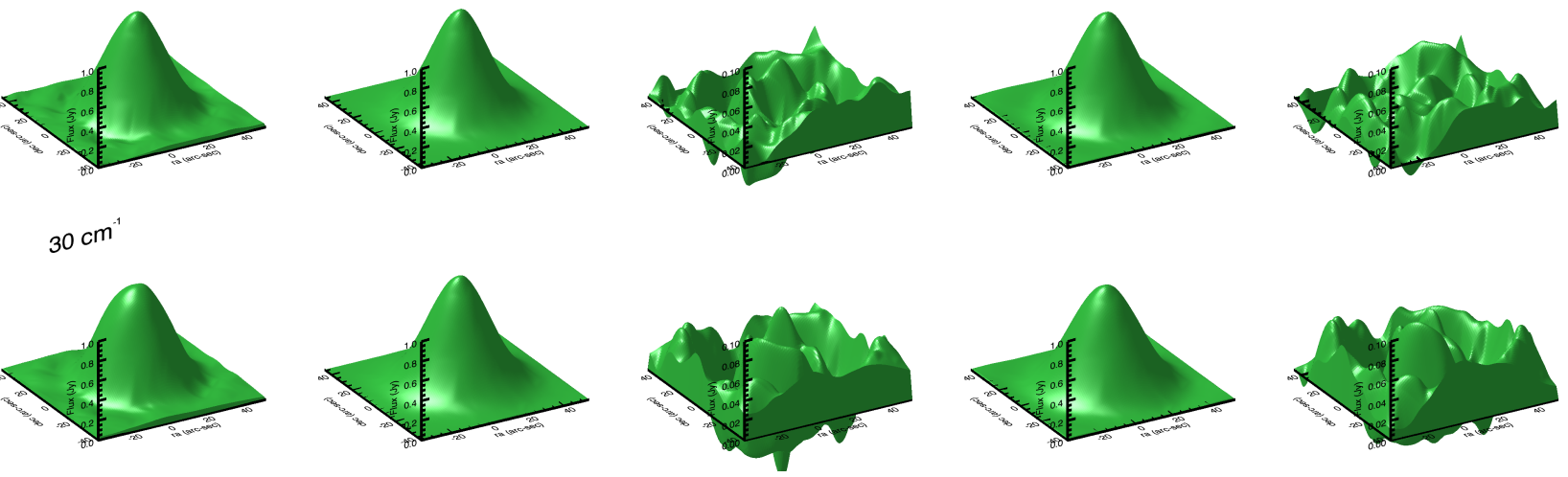

$40 \mathrm{~cm}$
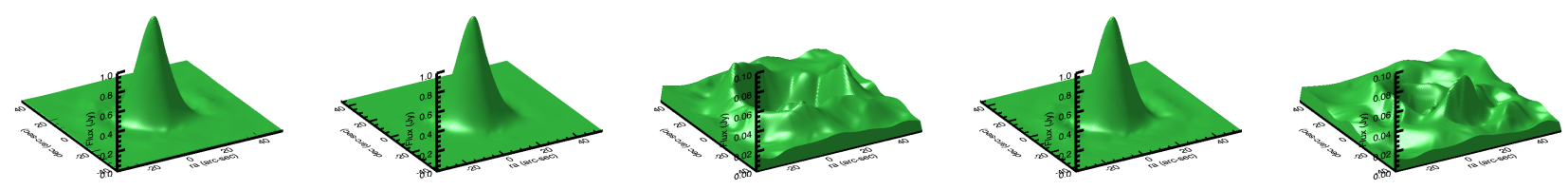

$50 \mathrm{~cm}$
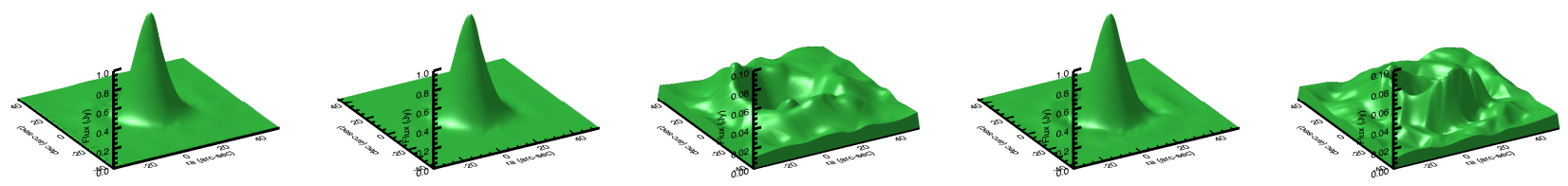

Figure 9. Normalized surface plots of the SPIRE FTS beam profile derived from scans of Neptune. The raw spectral data are shown in the left column at frequencies of 20,30, 40 and $50 \mathrm{~cm}-1$ respectively. The Gaussian and Airy elliptical best fits are shown in columns 2 and 4 with the respective residuals (x10) in columns 3 and 5 . The spatial scale extent of each plot is $1.5 \times 1.5$ arcminutes.

Table 3. Values of the FWHM in arcseconds of the best fit 2-dimensional Gaussian and Airy beam profiles to the data shown in Figure 10, at select frequencies. Also shown are the $1-\sigma$ errors and the ellipticity of the beams for both functions.

\begin{tabular}{|c|c|c|c|c|c|c|}
\hline \multirow{3}{*}{$\sigma\left(\mathrm{cm}^{-1}\right)$} & \multicolumn{3}{|c|}{ Gaussian Fit } & \multicolumn{3}{|c|}{ Airy Fit } \\
\hline & \multicolumn{2}{|c|}{ FWHM } & \multirow[t]{2}{*}{$\varepsilon$} & \multicolumn{2}{|c|}{ FWHM } & \multirow[t]{2}{*}{$\varepsilon$} \\
\hline & RA & DEC & & RA & DEC & \\
\hline 17 & $35.2 \pm 1.5$ & $36.1 \pm 1.6$ & 1.03 & $34.9 \pm 1.3$ & $36.0 \pm 1.3$ & 1.03 \\
\hline 20 & $31.7 \pm 0.3$ & $32.5 \pm 0.4$ & 1.03 & $33.3 \pm 0.3$ & $34.0 \pm 0.3$ & 1.02 \\
\hline 25 & $32.4 \pm 0.3$ & $33.8 \pm 0.3$ & 1.04 & $34.3 \pm 0.2$ & $35.7 \pm 0.2$ & 1.04 \\
\hline 30 & $34.6 \pm 0.5$ & $35.1 \pm 0.5$ & 1.02 & $37.0 \pm 0.4$ & $37.6 \pm 0.5$ & 1.02 \\
\hline 35 & $18.5 \pm 0.3$ & $19.4 \pm 0.3$ & 1.05 & $19.3 \pm 0.2$ & $20.3 \pm 0.3$ & 1.05 \\
\hline 40 & $17.0 \pm 0.3$ & $17.7 \pm 0.3$ & 1.04 & $17.6 \pm 0.2$ & $18.3 \pm 0.3$ & 1.04 \\
\hline 45 & $17.0 \pm 0.3$ & $17.7 \pm 0.3$ & 1.04 & $17.4 \pm 0.2$ & $18.3 \pm 0.3$ & 1.05 \\
\hline 50 & $16.7 \pm 0.3$ & $17.4 \pm 0.3$ & 1.04 & $17.1 \pm 0.2$ & $18.0 \pm 0.3$ & 1.05 \\
\hline
\end{tabular}




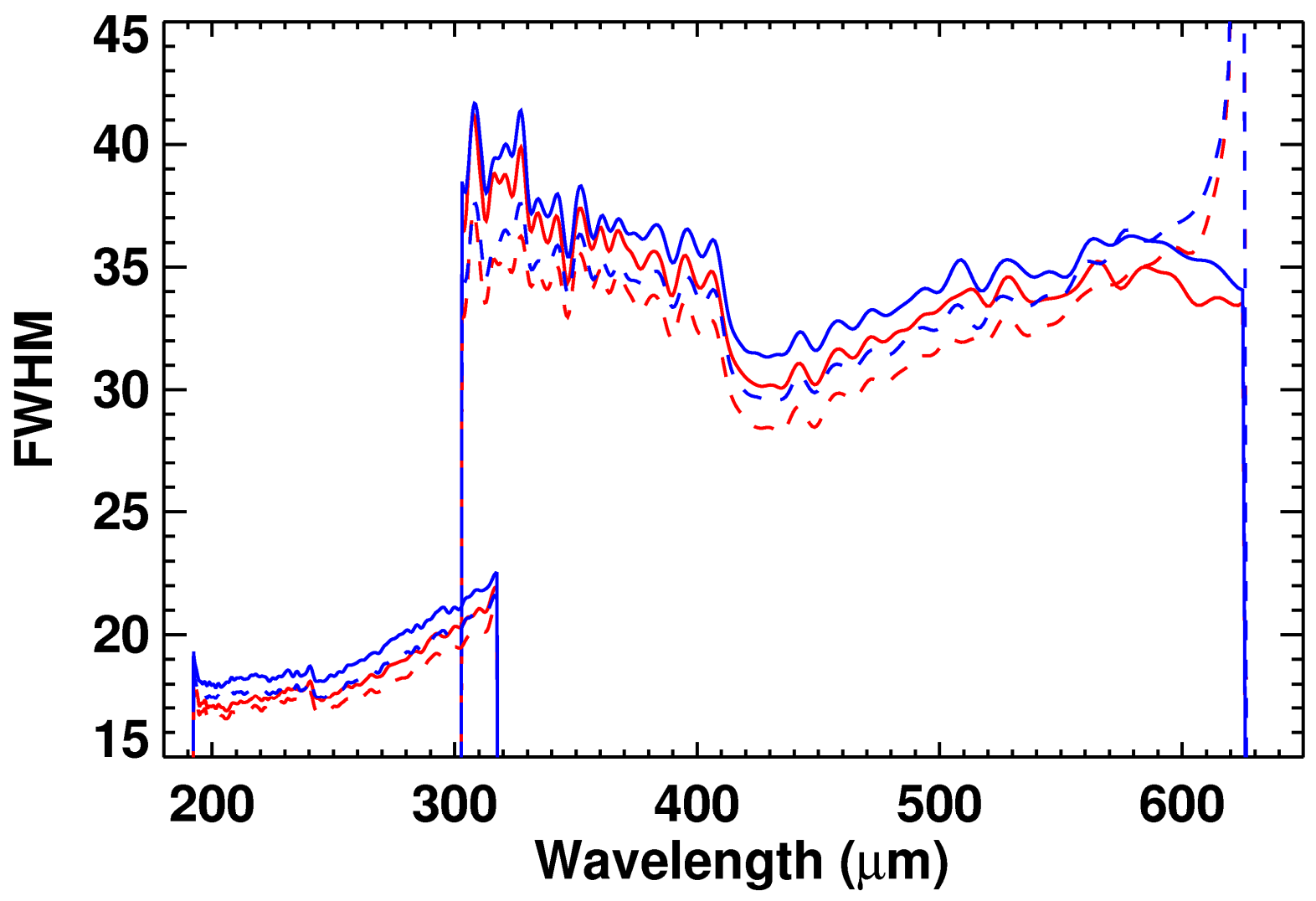

Figure 10. The wavelength dependent beam profiles (FWHM in arcseconds) derived.from fitting 2-dimensional Airy (solid lines) and Gaussian (dashed lines) to the Neptune scan data in Ra (red) and Dec (blue). Steps in the beam profile correspond to mode onset points of the feedhorns.

\subsection{Spectroscopic sensitivity}

The measured in-flight spectral line sensitivity when observing point sources, for using the three nominal spectroscopic observing modes (high, medium and low resolution) are summarized in Table 4. The line sensitivity achieved in the high-resolution mode of the FTS is $\sim 1.5 \times 10^{-17} \mathrm{Wm}^{-2}$ for the SSW band and $2.0 \times 10^{-17} \mathrm{Wm}^{-2}$ for the SLW band $(5 \sigma ; 1$ hr). The sensitivity varies slightly across the band as described in the SPIRE Observers Manual ${ }^{18}$. Analysis shows that the noise in the interferogram integrates down as the square root of the number of scans as expected. Since the Fourier transform is a linear transform, the noise in the spectrum should integrate down in a similar manner. Currently, while the spectral noise level does decrease with increasing number of scans, it does so at a rate that is slightly less than expected. Potential explanations for this effect include the bolometer detector non-linearity correction algorithm and the different thermal background seen by the detectors during the source and reference observations. In any astronomical observation, radiation from the source, the telescope, the second input port (SCal), and the instrument self emission are detected by the spectrometer bolometers. It is therefore necessary to subtract from the source interferogram a corresponding reference interferogram, taken on a suitable dark sky position. Differences in the radiant loading from any part of the instrument during the acquisition of the source and dark sky measurements will masquerade as erroneous spectral signatures in the recovered spectra.

Despite these issues, the FTS sensitivities are better than pre-launch predictions by a factor of $1.5-2$. This improvement is attributable to a combination of factors: the low telescope background, the fact that the SCAL source is not required to null the interferometer modulation at ZPD, the lower operating temperature of the detectors and the adoption of a conservatism factor that was applied to the modeled sensitivities to account for various uncertainties in the sensitivity model. 
Table 4. FTS point source sensitivity (unapodized) for the three nominal spectroscopic observing modes: high resolution (HR, $\Delta \sigma=$ $0.04 \mathrm{~cm}-1)$, medium resolution (MR, $\Delta \sigma=0.24 \mathrm{~cm}-1)$ and low resolution (LR, $\Delta \sigma=0.83 \mathrm{~cm}-1)$.

\begin{tabular}{|c|c|c|c|c|c|c|}
\hline \multirow[b]{2}{*}{ Band } & \multirow[b]{2}{*}{$\begin{array}{c}\sigma \\
\left(\mathrm{cm}^{-1}\right)\end{array}$} & \multirow[b]{2}{*}{$\begin{array}{c}\lambda \\
(\mu \mathrm{m})\end{array}$} & \multirow{2}{*}{ 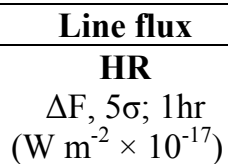 } & \multicolumn{3}{|c|}{ Continuum } \\
\hline & & & & $\begin{array}{l}\mathbf{H R} \\
\Delta \mathrm{S}, 5 \sigma ; 1 \mathrm{hr} \\
(\mathrm{Jy})\end{array}$ & $\begin{array}{c}\text { MR } \\
\Delta \mathrm{S}, 5 \sigma ; 1 \mathrm{hr} \\
\quad(\mathrm{Jy})\end{array}$ & $\begin{array}{c}\text { LR } \\
\Delta \mathrm{F}, \mathrm{S} \sigma ; 1 \mathrm{hr} \\
(\mathrm{Jy})\end{array}$ \\
\hline SLW & 31.9 & 313 & 1.70 & 1.42 & 0.24 & 0.068 \\
\hline SLW & 21.5 & 465 & 1.00 & 0.83 & 0.14 & 0.040 \\
\hline SLW & 14.9 & 671 & 2.20 & 1.83 & 0.31 & 0.088 \\
\hline SSW & 51.5 & 194 & 2.15 & 1.79 & 0.30 & 0.086 \\
\hline SSW & 50 & 200 & 2.00 & 1.67 & 0.28 & 0.080 \\
\hline SSW & 40 & 250 & 1.00 & 0.83 & 0.14 & 0.040 \\
\hline
\end{tabular}

\section{CONCLUSIONS AND FUTURE WORK}

The SPIRE spectrometer is fully functional with a performance matching or exceeding pre-launch estimates. In particular, measurements of the spectral range, instrumental line shape, wavelength accuracy, beam profile and sensitivity are in close agreement with either ground-based measurements, or where these were not available, theoretical models.

The current spectrometer pipeline ${ }^{19}$ is producing calibrated spectra of high quality. Initial spectral calibration was based on the asteroid Vesta, but is being updated to utilize the higher signal-to-noise measurements afforded by Uranus and Neptune using the planetary atmosphere models of Moreno ${ }^{18}$. Observations of a point source with the central detector pair (SLWC3/SSWD4) yield calibrated flux densities in Jy. For spectral mapping observations with full or intermediate spatial sampling, the pipeline produces calibrated, regularly gridded spectral cubes in units of intensity $\left(\mathrm{W} \mathrm{m}^{-2} \mathrm{sr}^{-1} \mathrm{~Hz}^{-1}\right)$ under the assumption of a uniformly extended source. Current flux calibration accuracy ${ }^{20}$ is estimated conservatively at $15-20 \%$ in the SSW band and $20-30 \%$ in the SLW band above $20 \mathrm{~cm}^{-1}$ and up to $50 \%$ below $20 \mathrm{~cm}^{-1}$.

The calibration procedures continue to be refined. The detector signal processing is being modified to operate in the more physically meaningful temperature domain, which should improve the non-linearity correction; an important first step in the data processing pipeline. Additional beam profile data have been obtained, which will allow a more detailed study of the wavelength dependent beam shape, which is important for the spectral mapping observations. Detailed modeling of the contributions from the telescope and instrument to the measured signal is being undertaken to account for the excess emission seen at frequencies below $25 \mathrm{~cm}^{-1}$. Finally, efforts are underway to remove the channel fringes, spectral features that arise from resonant optical cavities within the interferometer.

Notwithstanding these issues, the spectra produced by the SPIRE imaging Fourier transform spectrometer are of the highest quality which will allow astronomers to address some leading questions in modern astrophysics.

\section{ACKNOWLEDGMENTS}

SPIRE has been developed by a consortium of institutes led by Cardiff Univ. (UK) and including Univ. Lethbridge (Canada); NAOC (China); CEA, LAM (France); IFSI, Univ. Padua (Italy); IAC (Spain); Stockholm Observatory (Sweden); Imperial College London, RAL, UCL-MSSL, UKATC, Univ. Sussex (UK); Caltech, JPL, NHSC, Univ. Colorado (USA). This development has been supported by national funding agencies: CSA (Canada); NAOC (China); CEA, CNES, CNRS (France); ASI (Italy); MCINN (Spain); SNSB (Sweden); STFC (UK); and NASA (USA). DAN acknowledges support from the CSA and NSERC. The authors thank Brad Gom and Tanner Heggie for their contributions to the data analysis. 


\section{REFERENCES}

[1] G. Pilbratt et al., Herschel Space Observatory: An ESA facility for far-infrared and submillimetre astronomy, Astronomy and Astrophysics, in press, 2010.

[2] M. J. Griffin et al., The Herschel-SPIRE instrument and its in-flight performance, Astronomy and Astrophysics, in press, 2010.

[3] A. D. Turner, J. J. Bock, H. T. Nguyen et al., Silicon nitride Micromesh Bolometer Array for Submillimeter Astrophysics, Appl. Opt. 40, 4921-4932, 2001.

[4] B. Swinyard, K. Dohlen, D. Ferrand, et al., Imaging FTS for Herschel-SPIRE, Proc. SPIE 4850, 698-709, 2003.

[5] K. Dohlen, A. Origné, A., D. Pouliquen \& B. Swinyard, Optical design of the SPIRE instrument for FIRST, Proc. SPIE 4013, 119-128, 2000.

[6] P. Ade, G. Pisano, G., C. E. Tucker \& S. O. Weaver, A review of metal mesh filters, Proc. SPIE 6275, 62750U, 2006.

[7] P. C. Hargrave, J. W. Beeman, P. A. Collins, et al., In-flight calibration sources for Herschel-SPIRE, Proc. SPIE 4850, 638-649, 2003.

[8] D. A. Naylor, J-P Baluteau, P. Davis-Imhof, M. J. Ferlet, T. R. Fulton \& B. M. Swinyard Performance evaluation of the Herschel/SPIRE imaging Fourier transform spectrometer, Proc. SPIE 6265, 626530 (2006)

[9] T. de Graauw, F. P. Helmich, T. G. Phillips, et al., The Heterodyne Instrument for the Far Infrared (HIFI) on the Herschel Space Osbervatory, Astronomy and Astrophysics, in press, 2010.

[10] A. Poglitsch, C. Waelkens, N. Geis, et al. 2010, The Photodetector Array Camera and Spectrometer (PACS) on the Herschel Space Observatory, Astronomy and Astrophysics, in press, 2010.

[11] D. A. Naylor \& M. K. Tahic, Apodizing functions for Fourier transform spectroscopy, J. Opt. Soc. Am. A, 24, 3644-3648, 2007.

[12] L. D. Spencer, D. A. Naylor, B. Zhang, et al., Performance Evaluation of the Herschel/SPIRE Instrument Flight Model Imaging Fourier Transform Spectrometer, Proc. SPIE, 7010, 701008, 2008.

[13] E. Habart et al., SPIRE spectroscopy of the prototypical Orion Bar Photodissociation Region, Astronomy and Astrophysics, in press, 2010.

[14] L. D. Spencer, D. A. Naylor \& B. M. Swinyard, Performance evaluation of the Herschel/SPIRE imaging Fourier transform spectrometer through ground-based measurements, Meas. Sci. Technol. 21, 2010.

[15] D. A. Naylor, E. Dartois, E. Habart et al., First detection of the Methylidyne cation $\left(\mathrm{CH}^{+}\right)$fundamental rotational line with the Herschel/SPIRE-FTS, Astronomy and Astrophysics, in press, 2010.

[16] S. P. Davis, M. C. Abrams \& J. W. Brault, Fourier Transform Spectrometry, Academic Press, 2001.

[17] M. J. Griffin, J. J., Bock, \& W. K. Gear, Relative Performance of Filled and Feedhorn-Coupled Focal-Plane Architectures, Appl. Opt., 31, 6543-6554, 2002.

[18] SPIRE Observers' Manual, HERSCHEL-HSC-DOC-0789, version 2.0, May 2010 available at the ESA Science

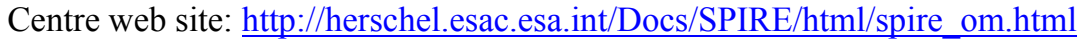

[19] T. R. Fulton, J-P Baluteau, G. Bendo et al., The data processing pipelines for the Herschel/SPIRE Imaging Fourier Transform Spectrometer, Proc. SPIE, 7731, in press, 2010.

[20] B. M. Swinyard et al., In-Flight Calibration of the Herschel-SPIRE Instrument, Astronomy and Astrophysics, in press, 2010. 